\title{
Analysis and Evaluation of Incentive-Compatible Dynamic Mechanisms for Carrier Collaboration
}

\author{
Miguel Andres Figliozzi
}

\begin{abstract}
This paper introduces a framework for carrier dynamic collaboration. In particular it proposes and analyzes dynamic collaborative mechanisms that are incentive compatible. The dynamic collaborative environment is characterized by a set of carriers that have a proprietary set of customers that generate a stream of random demands over time. The proposed collaborative mechanism is such that on each demand arrival each carrier has the incentive to submit the arrived shipment or service request to the collaborative mechanism. Intuition about the efficiency and the workings of the collaborative mechanism is developed. A general framework to formulate and study collaborative frameworks among transportation carriers is proposed. A truckload pickup-and-delivery collaborative environment is simulated, and results are analyzed.
\end{abstract}

Of current significance to the fields of logistics and supply chain is the concept of collaboration, enabled and partly driven by the extensive advances and changes in information and communication technologies that have taken place in the past few decades. For example, supply-chain collaborative processes such as collaborative planning forecasting and replenishment (1) make possible joint sales forecasting and replenishment planning between trading partners and enable participants to share improvements in inventory costs, revenue, and customer service levels.

Collaboration is not only an attempt to find win-win solutions to conflicting objectives but also an integration of behavioral, communicational, and interactive flows (1). Clearly, an important indicator of the viability of voluntary collaboration is the ability to find synergies that reduce operating costs. Strategic collaborations, effective coordination, and streamlined supply chain networks are key factors by which companies thrive in today's competitive business environment (2). In other instances, collaboration is induced by governmental regulation, as in the case of urban consolidation to reduce truck traffic in urban environments (3). Internet-based collaborative initiatives that aim at cost reduction through collaboration has spawned in recent years. For example, Nistevo (www.nistevo.com) is a web-based collaborative portal that allows shippers to reduce transportation costs; when shippers join their demands or requests, economies of scope or scale can be obtained. On the carrier side, web-based initiatives such as Transplace (www.transplace.com: a joint effort of six major USA-based carriers) aim at providing a common transactional point for shippers and carriers.

Institute of Transport and Logistics Studies, Faculty of Economics and Business, University of Sydney (C37), 144 Burren Street, Newtown, 2042, Australia.

Transportation Research Record: Journal of the Transportation Research Board, No. 1966, Transportation Research Board of the National Academies, Washington, D.C., 2006, pp. 34-40.
Collaboration brings about synergistic opportunities but also challenges as the problems grow in size and complexity. Rules and protocols are needed to regulate cooperative activity and to reduce unnecessary information overload and delays. This paper introduces a framework for carrier dynamic collaboration. In particular, it proposes and analyzes dynamic collaborative mechanisms that are incentive compatible. The dynamic collaborative environment is characterized by a set of carriers that have a proprietary set of customers that generate a stream of random demands over time. The proposed collaborative mechanism is such that on each demand arrival, each carrier has the incentive to submit the arrived shipment or service request to the collaborative mechanism.

Among the vast array of possible collaborative mechanisms with different payment, allocation, and trust structures (the term trust structures refers to the collection of policies used in a system to determine the trustworthiness of the participants), this research focuses on incentive compatible mechanisms. In incentive compatible mechanisms carriers submit (truthfully) only their cost estimations; they do not shade the value of their prices or bids' taking into account what the competition is likely to do. Such kind of mechanism has several advantages: $(a)$ costs are easier to compute than prices, $(b)$ the resulting mechanisms are conceptually simple and easy to understand and implement, and (c) carriers' best strategy is independent of the competition strategies. These advantages are extremely useful in a dynamic environment where price estimation problems can easily become intractable or computationally expensive. Carriers have the incentive to invest in technologies to reduce costs or better estimate them, or both. In addition, cost pricing is a more efficient auction mechanism than first-price pricing in a sequential transport marketplace (4).

\section{LITERATURE REVIEW}

A collaborative agreement cannot be established without clear rules that assure sustainability and ensure service fulfillment and control. Considerable effort is required to concur in an arrangement (collaborative rules) that satisfies the numerous participation and rationality constraints.

A collaborative outcome that benefits all parties is a necessary condition to facilitate collaboration, although this is a not a sufficient condition; this is clearly illustrated in the archetypical prisoner's dilemma (5). Carriers cannot be assumed to cooperate or sustain a collaborative outcome unless they have the incentive to do so. Unfortunately, in general it is impossible to simultaneously achieve perfect efficiency, budget balance [restricting the mechanism to be budget balanced precludes the use of external financial support (a subsidy) to sustain the operation of the collaborative mechanism], incentive 
compatibility, and individual rationality in a two-sided negotiation with self-interested agents, as shown by Myerson and Satterthwaite (6). Mechanisms based on marginal cost allocations such as the Vickrey-Clark-Grooves mechanism are efficient, individually rational, and incentive compatible, but not budget balanced (7). In general, it is impossible to obtain these four highly desirable conditions simultaneously.

The advent of the Internet has spurred the development of collaborative mechanisms mainly in the computer science literature. However, the particular characteristics of dynamic freight transportation systems preclude the direct transference of models from other fields (8). Computer science and supply-chain literature agent-based coordination models are reviewed in Sandholm (9) and $\mathrm{Wu}(10)$, respectively. Modeling approaches proposed in the artificial intelligence literature for freight transportation focus on decommitment strategies. Fischer et al. (11) and Burckert et al. (12) present a model where companies can break agreements at any time, at the cost of a renegotiated penalty, to take more profitable shipments. Decommitment simplifies calculations considerably but cannot guarantee meeting shipments time windows. In reality, decommitment is unacceptable for shippers who highly value on-time delivery performance (13). Decommitment is particularly unrealistic in just-in-time environments where penalties for late deliveries can exceed several times the cost of the service. Furthermore, a poor on-time-delivery record or excessive variability can lead to the nonrenewal of transportation contracts or even contract cancellations.

An electronic brokerage system for the trucking industry is proposed by Kim and Lee (14). The paper suggests an efficient auction-based method for matching delivery tasks with trucks. The problem is formulated from the point of view of the market maker but without analyzing incentive compatible, individual rationality, or budget balance issues. The closest work to this research is the paper by Song and Regan about an auction-based, postcontract, collaboration mechanism (15). In this work it is assumed that a group of carriers with overlapping service areas choose to collaborate; every time a carrier obtains a load he or she evaluates if the load is cost-effective to serve. If the load is not cost effective, the carrier estimates a reservation value and asks the other carriers to submit their bids. The shipment allocation or payment is completed using a second price auction, but the treatment of the problem is essentially static.

This research is different from previous research in several aspects: (a) the mechanism is dynamic; (b) the mechanism is incentive compatible; $(c)$ there is a detailed treatment of payment and information issues that ensure incentive-compatible constraints as well as the discussion of efficiencies; and $(d)$ simulation results of the mechanism are presented and analyzed.

\section{MARKET DESCRIPTION AND NOTATION}

The dynamic collaborative environment is characterized by a set of carriers that have a proprietary set of customers that generate a stream of random demands over time (In general, the mechanism proposed applies to any kind of dynamic demand steam or customer request that can be swapped among carriers without significantly affecting customer service. In particular, results obtained from the application of the mechanism to a truckload pickup-and-delivery service with time windows are discussed in the section Simulation of a SPDCM Bundle Size 1). The challenge for individual rationality collaborative mechanisms is to ensure that each carrier has the incentive to participate in the collaborative mechanism on each demand arrival.
The formulation presented below allows for a more precise discussion of the main issues in a dynamic collaborative mechanism. It is assumed that there are $n$ carriers collaborating using a collaborative mechanism $\mathcal{M}$; a carrier is denoted by $i \in \mathfrak{J}$ where $\mathfrak{\Im}=\{1,2, \ldots, n\}$ is the set of all carriers. Each carrier has its own set of customers that request transportation services dynamically. Let the arrival or request announcement epochs for carrier $i$ be $\left\{t_{1}^{i}, t_{2}^{i}, \ldots, t_{N}^{i}\right\}$ such that $t_{k}^{i}<$ $t_{k+1}^{i}$ and $t_{N}^{i}$ denotes the time of the last arriving shipment for carrier $i$. Let $S_{k}^{i}=\left\{s_{1}^{i}, s_{2}^{i}, \ldots, s_{k}^{i}\right\}$ be the set of arriving shipments for carrier $i$ up to time $t_{k}^{i}$, then $S_{N}^{i}=\left\{s_{1}^{i}, s_{2}^{i}, \ldots, s_{N}^{i}\right\}$. Arrival times and shipments are not known in advance. The arrival epochs $\left\{t_{1}^{i}, t_{2}^{i}, \ldots, t_{N}^{i}\right\}$ follow a general arrival process. Furthermore, arrival times and shipments are assumed to come from a probability space $\left(\Omega^{i}, \mathcal{F}^{i}, \mathcal{P}^{i}\right)$ with outcomes $\left\{\omega_{1}^{i}, \omega_{2}^{i}, \ldots, \omega_{N}^{i}\right\}$ where $\omega_{t}^{i}=\left\{t_{t}^{i}, s_{t}^{i}\right\}$.

The fleet status of carrier $i$ when shipment $s_{t}^{i}$ arrives is denoted as $z_{t}^{i}$, which comprises two sets: $\breve{S}_{t}^{i}$, which is the set of shipments held by carrier $i$ that are not fully served at time $t$, and $V_{t}^{i}$, which is the set of vehicles in the fleet of carrier $i$ [where the shipment attributes and origin, destination, time windows, penalties for late deliveries, etc.; and the vehicle attributes are current location, status (empty or loaded), driver constraints in hours per miles worked, etc.]. There is a state or assignment function such that at time $t$ the status of carrier $i$ is $z_{t}^{i}=\mathrm{a}^{i}\left(h_{t}, z_{t-1}^{i}\right)$. Let $h_{t}$ denote the history of allocations done through the collaborative mechanism up to but not including allocations at time $t$. Each carrier has a cost function $\mathrm{c}^{i}\left(s_{j}, z_{j}^{i}\right)$ that estimates the cost (incremental) of serving a new shipment $s_{j}^{i}$ when the status of the fleet is $z_{j}^{i}$.

Assuming deterministic travel times, the fleet status at a given time is a function of the previous fleet status, the history of collaboration, and the fleet management function $\mathrm{a}^{i}$. The history of collaborations $h_{t}$ is the time-based record of all shipment transfers among carriers up to time $t$. Each carrier $i$ has private information $\theta_{t}^{i}=\left(\mathrm{a}^{i}, \mathrm{c}^{i} \breve{S}_{t}^{i}, V_{t}^{i}\right.$, $\left.\Omega^{i}, \mathcal{F}^{i}, \mathcal{P}^{i}\right)$ at time $t$.

When an event triggers a collaborative call (usually a shipment arrival), carriers send private information $\vartheta_{t}^{i} \subseteq \breve{S}_{t}^{i}$ to the collaborative mechanism. For each possible element of $\vartheta_{t}^{i}$, the carrier submits a reservation value. For a shipment $s_{t}^{i}$, a reservation value $y\left(s_{t}^{i}\right) \in \mathbb{R}^{+}$ is a positive real number and denotes the maximum monetary value that a carrier is willing to pay to another carrier for servicing a shipment sent to the collaborative mechanism. For each element in $\vartheta_{t}^{i}$, each carrier submits a reservation cost $e\left(s_{t}^{i}\right)$. For a shipment $s_{t}^{i}$, a reservation cost $e\left(s_{t}^{i}\right) \in \mathbb{R}^{+}$is a positive real number that denotes the minimum monetary value that carrier $i$ is willing to charge another carrier for servicing a shipment sent to the collaborative mechanisms.

Reservation costs and values must include all relevant costs (including opportunity costs) associated with servicing (or not servicing) an additional shipment or shipments. It is assumed that all participating carriers compute these costs accurately. It is also assumed that if a carrier cannot meet the shipment delivery conditions (e.g., time windows), the submitted reservation cost is $e\left(s_{t}^{i}\right)=\infty$ or a sufficiently high number that is larger than the reservation value. It is assumed that carriers trigger a collaborative call as soon as a new request arrives.

\section{INCENTIVE-COMPATIBLE DYNAMIC COLLABORATIVE FRAMEWORK}

Herein, it is assumed that shipments, reservation values, and reservation costs are submitted and processed by the collaborative mechanism in real time in a first-in-first-out fashion. The proposed mechanism 
is called second-price-based dynamic collaborative mechanism (SPDCM). The term second price is used because the workings of the SPDCM are inspired by the workings of the one-item (static) second-price (OISP) auction. The OISP auction is incentive compatible and achieves perfect efficiency (7). This is possible because the monetary value (price) that the winning buyer pays is not influenced by the value of the winning bid. This key idea is used in the SPDCM to maintain incentive compatibility throughout the collaboration process. It is applied to the dynamic mechanism for Bundle Size 1 -where the collaborative mechanism requests reservation costs for at most one shipment per collaborative instance-and to the dynamic mechanism for Bundle Size 2-where the collaborative mechanism requests reservation costs for at most two shipments per collaborative instance and where a carrier can submit reservation costs for each individual shipment or the combined bundle. These dynamic mechanisms and their properties are described henceforth.

\section{Bundle Size 1}

When the bundle size is 1 , each carrier can submit at most one reservation cost, and only one carrier can submit a shipment to the collaborative mechanism. In the proposed SPDCM a carrier $i$ submits a just arrived shipment $s_{j}^{i}$ and its reservation value $y\left(s_{j}^{i}\right)$ to the collaborative mechanism. The other carriers, denoted by $-i$, independently submit reservation costs which form the set

$e^{-i}\left(s_{j}^{i}\right)=\left[e^{1}\left(s_{j}^{i}\right), \ldots, e^{i-1}\left(s_{j}^{i}\right), e^{i+1}\left(s_{j}^{i}\right), \ldots, e^{N}\left(s_{j}^{i}\right)\right]$

The set $e^{\Im}\left(s_{j}^{i}\right)$ is formed by adding the set of reservation costs and reservation values,

$e^{\Im}\left(s_{j}^{i}\right)=\left[e^{1}\left(s_{j}^{i}\right), \ldots, e^{i-1}\left(s_{j}^{i}\right), y\left(s_{j}^{i}\right), e^{i+1}\left(s_{j}^{i}\right), \ldots, e^{N}\left(s_{j}^{i}\right)\right]$

Let the ordered version of this set be denoted

$e^{(\mathfrak{\Im})}\left(s_{j}^{i}\right)=\left[e^{(1)}\left(s_{j}^{i}\right), \ldots, e^{(N)}\left(s_{j}^{i}\right)\right]$

where $e^{(1)}\left(s_{j}^{i}\right)$ denotes value of the lowest element of the set $e^{\Im}\left(s_{j}^{i}\right)$, $e^{(2)}\left(s_{j}^{i}\right)$ the second lowest value of the set $e^{\Im}\left(s_{j}^{i}\right)$, and so on until $e^{(M)}\left(s_{j}^{i}\right)$ represents the highest element of the set $e^{\Im}\left(s_{j}^{i}\right)$. The term $\left(s_{j}^{i}\right)^{(k)}$ refers to the carrier that occupies the $k$ th position, carrier $(k)$, in the ordered set $e^{(\Im)}\left(s_{j}^{i}\right)$. The collaborative mechanism works as follows:

1. Carrier $i$ submits a just arrived shipment $s_{j}^{i}$ and its private (secret) reservation value $y\left(s_{j}^{i}\right)$.

2. The collaborative mechanisms informs the other carriers (denoted by $-i$ ) of shipment $s_{j}^{i}$ arrivals.

3. The carriers independently submit reservation costs that will comprise $e^{-i}\left(s_{j}^{i}\right)$.

4. The collaborative mechanism forms the ordered set $e^{(\Im)}\left(s_{j}^{i}\right)$.

5. If $e^{(2)}\left(s_{j}^{i}\right)<y\left(s_{j}^{i}\right)$ or $y\left(s_{j}^{i}\right)=e^{(2)}\left(s_{j}^{i}\right)=e^{(3)}\left(s_{j}^{i}\right)$, then carrier (1) is going to serve the shipment $s_{j}^{i}$ and will be paid an amount equal to $e^{(2)}\left(s_{j}^{i}\right)$.

6. Otherwise, $e^{(2)}\left(s_{j}^{i}\right) \geq y\left(s_{j}^{i}\right)$, the shipment is served by carrier $i$.

\section{Bundle Size 1: Pricing Strategies and Properties}

The proposed SPDCM inherits the budget balance, individual rationality, and incentive compatible, properties of the second-price auction. The incentive compatible property is conserved because it is optimal for the carriers to submit their respective incremental service costs as reservation values and costs. Two distinct cases are possible: $(a)$ the carrier submits a shipment and reservation value and (b) the carrier submits a reservation cost. In the first case are three possible scenarios: $(a .1)$ the carrier submits a reservation value higher than his or her own service cost, $(a .2)$ the carrier submits a reservation value equal to his or her service cost, and (a.3) the carrier submits a reservation value lower than his or her own service cost. A submission as in $a .1$ cannot be optimal because the carrier creates the possibility of paying more than what it costs to serve the shipment himself or herself. A submission as in $a .3$ cannot be optimal because the carrier decreases the possibility of $e^{(2)}\left(s_{j}^{i}\right)<y\left(s_{j}^{i}\right)$ taking place and does not decrease his or her payment, which is $e^{(2)}\left(s_{j}^{i}\right)$. In Case $b$ are three possible scenarios: $(b .1)$ the carrier submits a reservation cost higher than his or her service cost, $(b .2)$ the carrier submits a reservation cost equal to his or her service cost, and (b.3) the carrier submits a reservation cost lower than his or her service cost. The same logic as in Case $a$ applies. A submission as in $b .1$ cannot be optimal because the carrier decreases the possibility of being carrier (1) without changing payment value $e^{(2)}\left(s_{j}^{i}\right)$. A submission as in $b .3$ cannot be optimal because the carrier submits a reservation cost that is lower than his or her own service cost. Therefore, submitting reservation values and costs equal to the service costs is a weakly dominant strategy.

The budget balance and individual rationality properties are maintained because no carrier ever pays more than his or her reservation value. The mechanism is individual rationality because reservation costs and reservation values are always respected; no carrier is forced to receive less or pay more than what he or she is willing to accept. The mechanism is also budget balanced since payments never exceed the reservation value of the carrier offering the shipment to the collaborative mechanisms.

Full efficiency cannot be guaranteed in a dynamic environment as in a one-item auction. There are two sources of inefficiencies: $(a)$ the necessity to keep incentive compatible property and $(b)$ the impossibility of ensuring ex post efficiency (dynamic problem). The former type of inefficiency takes place when $e^{(1)}\left(s_{j}^{i}\right)<y\left(s_{j}^{i}\right)=e^{(2)}\left(s_{j}^{i}\right)<e^{(3)}\left(s_{j}^{i}\right)$; since $e^{(3)}\left(s_{j}^{i}\right)>y\left(s_{j}^{i}\right)$ and $e^{(2)}\left(s_{j}^{i}\right)=y\left(s_{j}^{i}\right)$, the shipment is served by carrier $i$ (as indicated by Item 6 of SPDCM). In this case it would have been more efficient if carrier $\left(s_{j}^{i}\right)^{(1)}$ had served the shipment. This type of inefficiency is necessary to ensure that the monetary value (price) paid to the winning carrier is not influenced by the value of the winning bid or the reservation value. Otherwise, if carrier $i$ knows that the payment is somehow dependent on his or her reservation value, he or she will be tempted to shade his submission and violate the incentive compatible condition. This type of consideration was first hinted by Vickrey (16) in his seminal paper about the workings of first and second price auctions and more recently employed by Ausubel and Cramton (17) to maintain efficiency in a multiunit second-price auction.

The latter type of inefficiency may take place even when $e^{(1)}\left(s_{j}^{i}\right)<$ $e^{(2)}\left(s_{j}^{i}\right)<y\left(s_{j}^{i}\right)$ and the shipment is assigned to carrier $\left(s_{j}^{i}\right)^{(1)}$. This assignment is ex ante efficient (on the basis of the cost expectations that each individual carriers have at time $t_{j}$ ), but in general it cannot be guaranteed that this would be an ex post efficient assignment. This type of inefficiency is common to all stochastic systems because it would be avoided only if future realizations could be flawlessly predicted.

The objective of the SPDCM is to maximize system profit in the collaborative mechanisms subject to budget balance, individual rationality, and incentive compatible constraints. This is done in Item 5 with 
the assignment of the shipment to the carrier with the lowest price. The generated system profit is denoted as $\sigma\left(s_{j}^{i}\right)=y\left(s_{j}^{i}\right)-e^{(1)}\left(s_{j}^{i}\right)$. The system profit maximization problem can be expressed as

$\max \sigma\left(s_{j}^{i}\right) x_{j}$

subject to

$$
y\left(s_{j}^{i}\right) \geq e^{(2)}\left(s_{j}^{i}\right) x_{j}
$$

and

$x_{j} \in\{0,1\}$

where $x_{j}$ is the only decision variable. If $x_{j}=1$, shipment $s_{j}^{i}$ is allocated to carrier (1) who pays $e^{(2)}\left(s_{j}^{i}\right)$ to carrier $i$; if $x_{j}=0$, shipment $s_{j}^{i}$ is served by carrier $i$.

\section{Bundle Size 2}

In this case, the collaborative mechanism collects reservation values and costs for up to two shipments (including possible bundles) and selects the best packing or allocation of carriers to shipments. If several shipments are offered simultaneously, a problem similar to a combinatorial auction is brought about. However, the dynamic aspects of the problem significantly change the implementation of the collaborative mechanism.

Let $S_{k}=\left\{s_{1}, s_{2}, \ldots, s_{k}\right\}=\cup_{i \in \Im} S_{k}^{i}$ be the set of all arriving shipments to the carriers that participate in the collaborative mechanism, arranged in ascending order of arrival and up to time $t_{k+1}$ but not including shipment $s_{k+1}$. Without loss of generality, assume that the first arrivals are for carriers $i$ and $j$, respectively. The collaborative mechanism bundles the shipments so $\vartheta_{1}^{\Im}=\varnothing$ (no action taken at time $t_{1}$ ) and $\vartheta_{2}^{\Im}=\left\{s_{1}, s_{2}\right\}$, where $s_{1}^{i}=s_{1}, s_{1}^{j}=s_{2}$. By defining

$$
\begin{aligned}
& \sigma\left(s_{1}\right)=y\left(s_{1}\right)-e^{(1)}\left(s_{1}\right) \\
& \sigma\left(s_{2}\right)=y\left(s_{2}\right)-e^{(1)}\left(s_{2}\right) \\
& \sigma\left(s_{1}, s_{2}\right)=y\left(s_{1}\right)+y\left(s_{2}\right)-e^{(1)}\left(s_{1}, s_{2}\right)
\end{aligned}
$$

the system profit maximization problem becomes

$\max \sigma\left(s_{1}\right) x_{1}+\sigma\left(s_{2}\right) x_{2}+\sigma\left(s_{1}, s_{2}\right) x_{12}$

subject to

$$
\begin{aligned}
& y\left(s_{1}\right) \geq e^{(2)}\left(s_{1}\right) x_{1} \\
& y\left(s_{2}\right) \geq e^{(2)}\left(s_{2}\right) x_{2} \\
& y\left(s_{1}\right)+y\left(s_{2}\right)>\min \left[e^{(2)}\left(s_{1}, s_{2}\right), e^{(2)}\left(s_{1}\right)+e^{(2)}\left(s_{2}\right)\right] x_{12} \\
& x_{1}+x_{12} \leq 1 \\
& x_{2}+x_{12} \leq 1 \\
& \text { and }
\end{aligned}
$$

$$
x_{1}, x_{2}, x_{12} \in\{0,1\}
$$

The SPDCM works as follows:

1. Carrier $i$ submits a just arrived shipment $s_{1}$ at time $t_{1}$ and indicates that it is willing to wait up to time $t_{1+}$ (time deadline) to form a bundle.

2. The collaborative mechanism keeps the information and does not inform the other carriers about it.

3 . If the next shipment does not arrive before the deadline $t_{1+}<$ $t_{1}^{j}=t_{2}$, there is no possibility of bundling. Shipment $s_{1}^{i}$ is offered by the collaborative mechanism at time $t_{1+}$ as a bundle of size 1 .

4. If $t_{1+} \geq t_{1}^{j}=t_{2}$, then at time $t_{2}$ carrier $i$ is asked by the collaborative mechanism to submit $y\left(s_{1}^{i}\right)=y\left(s_{1}\right)$, and carrier $j$ submits $s_{1}^{j}=$ $s_{2}, t_{2+}$ and $y\left(s_{1}^{j}\right)=y\left(s_{2}\right)$.

5. The collaborative mechanism forms $y\left(s_{1}, s_{2}\right)=y\left(s_{1}^{i}\right)+y\left(s_{1}^{j}\right)$.

6. At time $t_{2}$, the collaborative mechanism informs

a. The other carriers (all but carrier $i$ and $j$ ) of shipments

$\left\{s_{1}, s_{2}\right\}$ arrivals,

b. Carrier $i$ about shipment $\left\{s_{2}\right\}$, and

c. Carrier $j$ about shipment $\left\{s_{1}\right\}$.

7. The carriers independently submit reservation costs:

a. Each of the carriers $k \in \mathfrak{\Im}$ such that $k \neq i, k \neq j$ submits three reservation costs: $e^{k}\left(s_{1}, s_{2}\right), e^{k}\left(s_{1}\right)$, and $e^{k}\left(s_{2}\right)$.

b. Carrier $i$ submits reservation costs $e^{i}\left(s_{2}\right)$ and $e^{i}\left(s_{1}, s_{2}\right)$.

c. Carrier $j$ submits reservation costs $e^{j}\left(s_{1}\right)$ and $e^{j}\left(s_{1}, s_{2}\right)$.

8. The collaborative mechanism forms the ordered sets $e^{(\Im)}\left(s_{1}\right)$, $e^{(\Im)}\left(s_{2}\right)$, and $e^{(\Im)}\left(s_{1}, s_{2}\right)$.

9. The collaborative mechanism maximizes system profits (optimize system profits using decision variables $x_{1}, x_{2}, x_{12}$ ).

10. Allocations and payments depends on the values of $x_{1}, x_{2}, x_{12}$ : a. If $x_{1}=1$, then carrier $\left(s_{1}\right)^{(1)}$ - the carrier with the lowest rate for $s_{1}$-is going to serve shipment $s_{1}$ and will be paid an amount equal to min $\left[e^{-*(1)}\left(s_{1}, s_{2}\right)-e^{(1)}\left(s_{2}\right) x_{2}, e^{(2)}\left(s_{1}\right)\right]$. The notation -* is used to indicate that the winner, the carrier that submitted $e^{(1)}\left(s_{1}\right)$, is excluded from the sets that may determine the payment level.

b. If $x_{2}=1$, then carrier $\left(s_{2}\right)^{(1)}$ - the carrier with the lowest rate for $s_{2}$-is going to serve shipment $s_{2}$ and will be paid an amount equal to $\min \left[e^{-*(1)}\left(s_{1}, s_{2}\right)-e^{(1)}\left(s_{1}\right) x_{1}, e^{(2)}\left(s_{2}\right)\right]$. The notation -* is used to indicate that the winner, the carrier that submitted $e^{(1)}\left(s_{2}\right)$, is excluded from the sets that may determine the payment level.

c. If $x_{12}=1$, then carrier $\left(s_{1}, s_{2}\right)^{(1)}$ - the carrier with the lowest rate for the bundle $\left\{s_{1}, s_{2}\right\}$-is going to serve shipments $\left\{s_{1}, s_{2}\right\}$ and will be paid an amount equal to $\min \left[e^{(2)}\left(s_{1}, s_{2}\right), e^{-*(1)}\left(s_{1}\right)+\right.$ $\left.e^{-*(1)}\left(s_{2}\right)\right]$. The notation -* is used to indicate that the winner, the carrier that submitted $e^{(1)}\left(s_{1}, s_{2}\right)$, is excluded from the sets that may determine the payment level.

d. If $x_{1}=0$ and $x_{12}=0$, carrier $i$ will serve $s_{1}$.

e. If $x_{2}=0$ and $x_{12}=0$, carrier $j$ will serve $s_{2}$.

\section{Bundle Size 2: Pricing Strategies and Properties}

This extension conserves the individual rationality, incentive compatible, and budget balance properties. In all cases a carrier payment cannot be influenced by its reservation value or cost submissions. As before, submitting service costs is a weakly dominant strategy. Participation and submission of arriving shipments is individual rationality because the reservation value is not determined until the moment of the matching, and carriers will never pay anything above the reservation value or serve any shipment whose cost is less than the payment received. To keep incentive compatible, it is necessary to keep the submission of the first shipment of the bundle secret. Otherwise, for the second arriving shipment the second carrier can 
use this knowledge to determine the price of the bundle and increase the reservation value for the second arriving shipment. For a similar reason, after the collaborative mechanism has informed all carriers about a shipment arrival (the information is common knowledge), a shipment that was not assigned in the first attempt must be served by the initial carrier (Item 10.d or 10.e). As in the case of a Bundle Size 1, the incentive compatibility constraints that are needed to simplify the carriers' estimation problem can potentially reduce the efficiency of the collaborative mechanism allocations.

Dynamic bundling can exploit complementarities among arriving shipments, but it also increases the size of the system profit maximization problem and carrier calculations exponentially with the size of the bundle. Not only does the number of possible bundles grow exponentially with the number of shipments, but also each cost estimation is in general an NP-hard (nondeterministic polynomialtime hard) problem (18). Further, the intricacy of the cost or price estimation problem grows considerably in a dynamic stochastic setting $(19,20)$.

In a dynamic setting it is important not only that the size of the problem or computation time not grow exponentially but also that the waiting time to form a bundle not be too long. For example, the carrier submitting the first shipment has a trade-off between waiting longer to form a bundle (potential obtainment of savings) and serving the shipment early to increase carrier serving capacity. Carriers that submit shipments hoping to form a bundle must estimate a $t_{j+}$ deadline time. In a deterministic environment, this time is lower bounded by the arrival time and upper bounded by the latest pickup and delivery time and the location/status of the fleet.

In the SPDCM with a bundle size of 2, there is a probability of allocating single shipments (Bundle Size 1) when, for example, $t_{1+}<t_{1}^{j}=t_{2}$. It can be speculated that as the shipment arrival rate decreases or the shipment time windows narrow, the probability of forming larger shipments decreases. If the allowed bundle size is further increased, trade-offs are likely to be found between potential bundling efficiencies, the complexity of the collaborative mechanism, and the probability of forming large bundles (the average bundle size of the collaborative mechanism depends on the characteristics of the demand such as arrival rate and time windows).

\section{SIMULATION OF A SPDCM BUNDLE SIZE 1}

To measure the savings that can be obtained using a SPDCM, a hypothetical square geographic region with four identical carriers is simulated. It is assumed that the length of the square sides is equal to 1 unit of distance. For convenience, trucks travel at a constant speed equal to one unit of distance per unit of time. Demands for truckload pickup-and-delivery arise over this area and over time. Origins and destinations of demands are uniformly distributed over the square area, so the average loaded distance for a request is 0.52 unit of distance. All the arrivals are random; the arrival process follows a time Poisson process. The expected interarrival time for each carrier is $E[T]=1 /(K \lambda)$, where $\lambda$ is the demand request rate per vehicle and $K=2$ for each carrier. The arrival rate is the same for all carriers but the arrival times are simulated using a different random seed for each carrier. Three different Poisson arrival rates per truck per unit of time are simulated:

- $\lambda=0.5$ (uncongested),

- $\lambda=1.0$ (congested), and

- $\lambda=2.0$ (extremely congested).
The shipments have hard time windows. Three different time windows are simulated. In all cases, it is assumed that the earliest pickup time is the arriving time of the demand to the marketplace. The latest delivery times (LDT), in an order that reflects increasing slackness, are the following:

- LDT1 $=$ arrival time $+\mathbf{1} \times($ shipment loaded distance +0.25$)+$ $1 \times$ uniform $(0.0,1.0)$,

- LDT2 $=$ arrival time $+\mathbf{2} \times($ shipment loaded distance +0.25$)+$ $2 \times$ uniform $(0.0,1.0)$, and

- LDT3 $=$ arrival time $+\mathbf{4} \times($ shipment loaded distance +0.25$)+$ $4 \times$ uniform $(0.0,1.0)$.

The respective time windows (TWs) are called TW1, TW2, and TW3. The first type of time windows (TW1) provides hardly enough flexibility of scheduling. The opposite can be said about the last type (TW3). It is also assumed that all the vehicles and loads are compatible; no special equipment is required for specific loads. In all the simulations, carriers estimate service costs using an optimal static formulation developed by Yang et al. (21). All shipments have a reservation value distributed as uniform $(1.42,1.52)$. In all cases, reservation values exceed the maximum static incremental cost possible (therefore highest bid) in the simulated area $(\approx 1.41$ units of distance).

Three different performance measures are used to evaluate the performance of the four carrier systems with and without SPDCM. The first is the carrier's average empty distance or the average distance from the destination of one load to the origin of the next load served. Average empty distance is a measure of the allocation efficiency of the collaborative mechanism. The second performance measure is the number of shipments served. The third is the sum of individual carrier profits (which equals for each carrier the sum of all served shipments shipper reservation values minus the distance incurred to serve them all). The first performance measure is related to the efficiency of the carriers, the second one is related to the efficiency of the mechanism, and the third one to the efficiency and performance of the mechanism.

\section{ANALYSIS OF RESULTS}

Figures 1 to 3 show the changes in average empty distance, total number of served shipments, and total system profit generated when the SPDCM is implemented. The system without SPDCM is used as a base for comparison. Figure 1 indicates that deadheading is reduced considerably across the board, ranging from $24 \%$ to nearly $50 \%$. The number of served shipments increased considerably with short TWs and at high arrival rates (ARs) (Figure 2). The collaborative system clearly generates more system profit than a system of independent shipper-carrier pairs. In absolute terms, the additional system profit that is generated by the marketplace increases with the arrival rate. However, percentagewise, the major increases correspond to short time windows and, in a lesser degree, to high ARs. Collaboration is increasing the system's capacity in constrained environments or with high arrival rates (Figures 2 and 3).

\section{DISCUSSION OF RESULTS}

The collaborative mechanism clearly outperforms the system when carriers act individually in the stylized simulation market. However, important assumptions were made that can be difficult to match in 


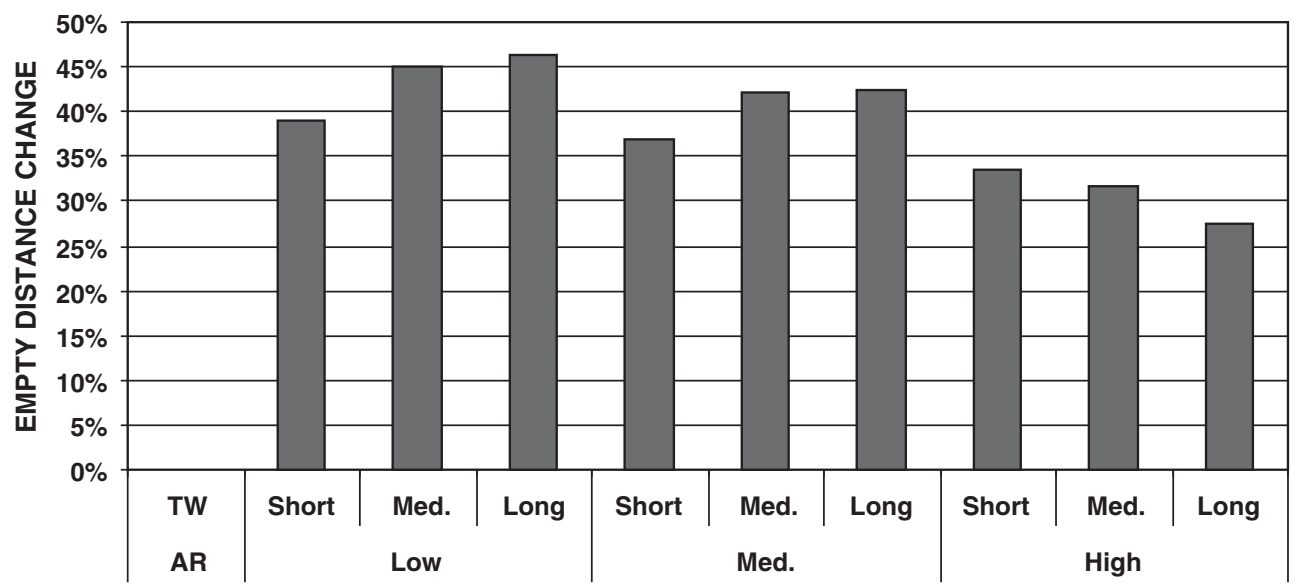

FIGURE 1 Average empty-distance improvement against base case (no collaboration).

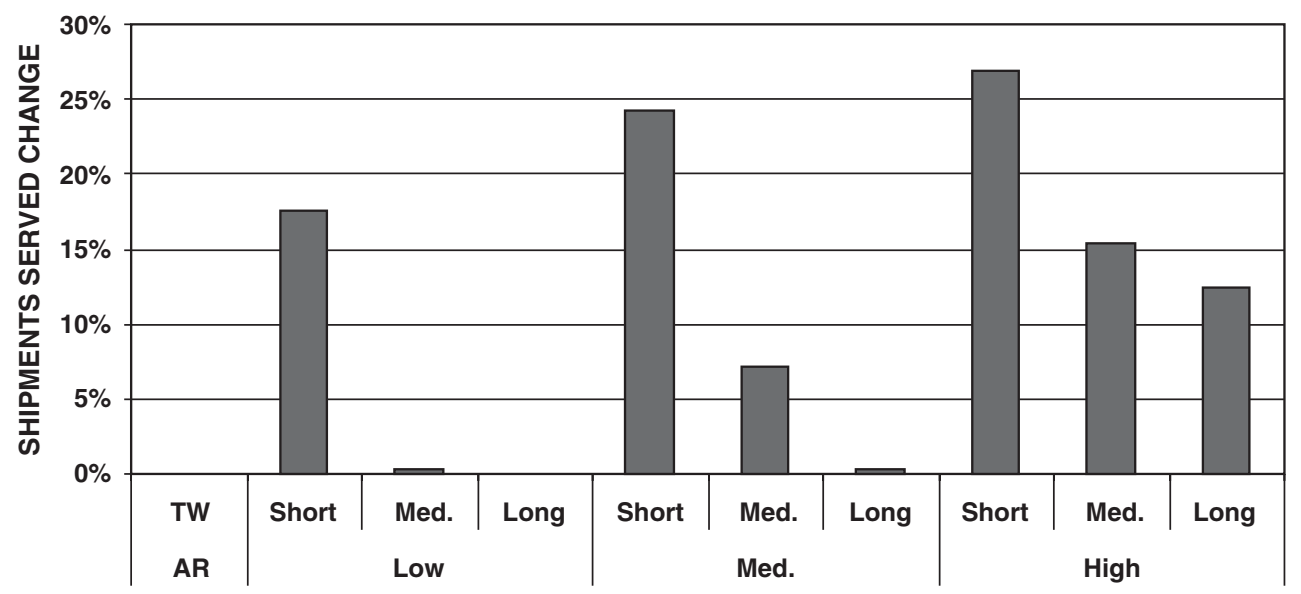

FIGURE 2 Shipment-served improvement against base case (no collaboration).

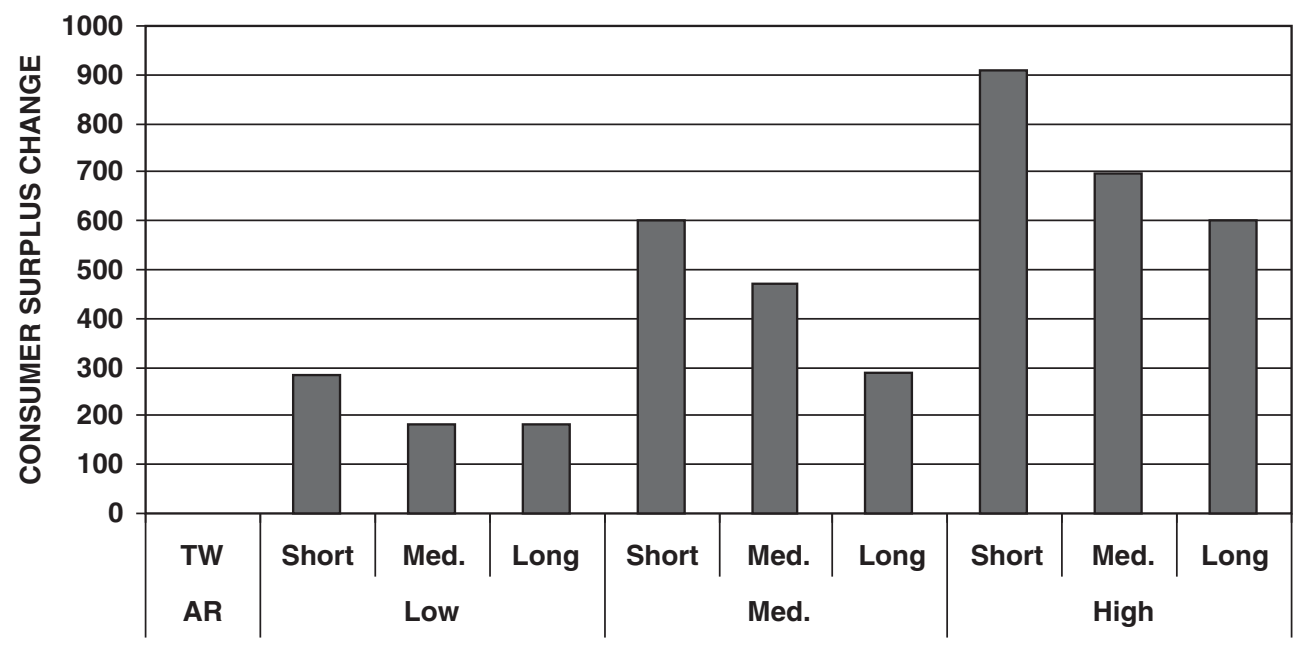

FIGURE 3 Total system profit improvement against base case (no collaboration). 
practice: $(a)$ customers do not object to being delivered by a truck or driver from a different company, $(b)$ all the vehicles and loads are compatible, $(c)$ collaborative mechanism implementation and operation costs are not considered, $(d)$ implementation of the collaborative mechanism and communications among carriers is flawless and in real-time, and $(e)$ the service areas of the carriers overlap completely. However, it is also clear that in some cases there could be substantial benefits from dynamic collaboration. Especially, when each individual carrier has a substantial amount of empty miles and the service areas overlap. Further research is needed to understand how shipment bundle size affects the efficiency of the system.

\section{CONCLUSIONS}

This paper introduced a dynamic collaborative mechanism that is incentive compatible. This has clear advantages for participating carries since only service costs must be estimated, which avoids price estimation problems that can easily become intractable or computationally expensive.

Dynamic bundling can exploit complementarities among arriving shipments, but it also increases the size of the system profit maximization problem and the number of carrier calculations exponentially with the size of the bundle. Not only does the number of possible bundles grow exponentially with the number of shipments but cost estimation also becomes more difficult. Simulation results have shown that the collaborative mechanism can easily outperform a noncollaborative system when carriers' service areas overlap and truck and loads can be easily swapped among carriers without affecting customer service requirements.

\section{ACKNOWLEDGMENT}

This project was partially funded by a grant from the Business School of the University of Sydney, Australia.

\section{REFERENCES}

1. Esper, T. L., and L. R. Williams. The Value of Collaborative Transportation Management (CTM): Its Relationship to CPFR and Information Technology. Transportation Journal, Vol. 42, No. 4, 2003, pp. 55-65.

2. Kelle, P., and A. Akbulut. The Role of ERP Tools in Supply Chain Information Sharing, Cooperation, and Cost Optimization. International Journal of Production Economics, Vol. 93-94, 2005, pp. 41-52.

3. Nemoto, T. Area-Wide Inter-Carrier Consolidation of Freight in Urban Areas. Transport Logistics, Vol. 12, No. 2, 1997, pp. 87-101.

4. Figliozzi, M. A., H. S. Mahmassani, and P. Jaillet. Impact of Auction Settings on the Performance of Truckload Transportation Marketplaces.
In Transportation Research Record: Journal of the Transportation Research Board, No. 1906, Transportation Research Board of the National Academies, Washington, D.C., 2005, pp. 89-96.

5. Axelrod, R., and W. D. Hamilton. The Evolution of Cooperation. Science, Vol. 211, 1981, pp. 1390-1396.

6. Myerson, R. B., and M. A. Satterthwaite. Efficient Mechanisms for Bilateral Trading. Journal of Economic Theory, Vol. 29, No. 2, 1983 , pp. 265-281.

7. Krishna, V. Auction Theory. Academic Press, San Diego, Calif., 2002.

8. Figliozzi, M. A., H. S. Mahmassani, and P. Jaillet. Framework for Study of Carrier Strategies in Auction-Based Transportation Marketplace. In Transportation Research Record: Journal of the Transportation Research Board, No. 1854, Transportation Research Board of the National Academies, Washington, D.C., 2003, pp. 162-170.

9. Sandholm, T. Agents in Electronic Commerce: Component Technologies for Automated Negotiation and Coalition Formation. Autonomous Agents and Multi-Agent Systems, Vol. 3, No. 1, 2000, pp. 73-96.

10. Wu, D. J. Software Agents for Knowledge Management: Coordination in Multi-Agent Supply Chains and Auctions. Expert Systems With Applications, Vol. 20, No. 1, 2001, pp. 51-64.

11. Fischer, K., J. P. Muller, and M. Pischel. Cooperative Transportation Scheduling: An Application Domain for DAI. Journal of Applied Artificial Intelligence, Vol. 10, No. 1, 1996, pp. 1-33.

12. Burckert, H. J., K. Fischer, and G. Vierke. Holonic Transport Scheduling with TELETRUCK. Applied Artificial Intelligence, Vol. 14, No. 7, 2000, pp. 697-725.

13. Brooks, M. R. Performance Evaluation in the North American Transport Industry: Users' Views. Transport Reviews, Vol. 18, No. 1, 1998, pp. 1-16.

14. Kim, K., and J. Lee, An Auction-Based Dispatching Method for an Electronic Brokerage of Truckload Vehicles. Technical report. Department of Industrial Engineering, Pusan National University, Busah, South Korea, 2004.

15. Song, J., and A. C. Regan. An Auction Based Collaborative Carrier Network. Presented at 83rd Annual Meeting of the Transportation Research Board, Washington, D.C., 2004.

16. Vickrey, W. Counterspeculation, Auctions and Competitive Sealed Tenders. Journal of Finance, Vol. 16, 1961, pp. 8-37.

17. Ausubel, L. M., and P. Cramton. Vickrey Auctions with Reserve Pricing. Economic Theory, Vol. 23, No. 3, 2004, pp. 493-505.

18. Song, J., and A. Regan. Combinatorial Auctions for Trucking Service Procurement: An Examination of Carrier Bidding Policies. Presented at 10th International Conference on Travel Behaviour Research (IATBR), August 2003.

19. Figliozzi, M. A. Performance and Analysis of Spot Truck-Load Procurement Markets Using Sequential Auctions. PhD Thesis, School of Engineering, University of Maryland, College Park, 2004.

20. Figliozzi, M. A., H. S. Mahmassani, and P. Jaillet. Quantifying Opportunity Costs in Sequential Transportation Auctions for Truckload Acquisition. In Transportation Research Record: Journal of the Transportation Research Board, No. 1964, Transportation Research Board of the National Academies, Washington, D.C., 2006, pp. 247-252.

21. Yang, J., P. Jaillet, and H. Mahmassani. Real-Time Multivehicle Truckload Pickup and Delivery Problems. Transportation Science, Vol. 38, No. 2, 2004, pp. 135-148.

The Freight Transportation Planning and Logistics Committee sponsored publication of this paper. 